[4] Botson J and Peterson J. Ann Rheum Dis. 2019; 78: A1289

[5] Bessen SY, et al. Semin Arthritis Rheum. 2019;49:56-61.

[6] Berhanu AA, et al. Semin Arthritis Rheum. 2017;46:754-758.

Disclosure of Interests: : John Albert Consultant of: Horizon Therapeutics, Speakers bureau: Horizon Therapeutics, Tony Hosey Shareholder of: Horizon Therapeutics, Employee of: Horizon Therapeutics, Brian LaMoreaux Shareholder of: Horizon Therapeutics, Employee of: Horizon Therapeutics DOI: 10.1136/annrheumdis-2020-eular.2076

\section{THU0433 TREATMENT WITH PEGLOTICASE IMPROVES HEPATIC FIBROSIS ESTIMATED BY FIBROSIS-4 INDEX IN SUBJECTS WITH CHRONIC REFRACTORY GOUT}

N. Schlesinger ${ }^{1}$, A. Yeo ${ }^{2}$, P. Lipsky ${ }^{3}{ }^{1}$ Rutgers Robert Wood Johnson Medical School, New Brunswick, United States of America; ${ }^{2}$ Horizon Therapeutics, Lake Forest, United States of America; ${ }^{3}$ AMPEL BioSolutions, LLC, Charlottesville, United States of America

Background: Hyperuricemia is associated with non-alcoholic fatty liver disease (NAFLD) $)^{1,2}$, but the relationship to fibrosis remains uncertain ${ }^{3}$. Moreover, it is not known whether lowering serum urate will affect the course of NAFLD. The availability of data from two randomized trials of pegloticase, a pegylated recombinant mammalian uricase, that profoundly decreases serum urate afforded the opportunity to test the hypothesis that lowering urate might improve NAFLD.

Objectives: To determine whether treatment of chronic refractory gout patients with pegloticase was associated with improvement in NAFLD determined by Fibrosis 4 index (Fib4).

Methods: Databases from patients with chronic refractory gout who participated in two randomized 6 month clinical trials (RCTs) of pegloticase were analyzed ${ }^{4}$. Sub-sets who had persistent urate lowering to levels $<1 \mathrm{mg} / \mathrm{dL}$ in response to biweekly pegloticase (Responders, $n=36$ ) were compared to those who received placebo $(n=43)$. Since liver biopsy information was not available on these subjects, we relied on Fib4, a validated non-invasive estimate of liver fibrosis in a variety of liver diseases ${ }^{5,6}$ calculated from measurements of AST, ALT, platelet count and age (Age $\times$ AST/platelets $\times \sqrt{ }$ ALT). A Fib4 value of 1.3 is an indication that further evaluation of liver disease is warranted.

Results: At baseline, the mean Fib4 values were $1.40 \pm 0.86$ in pegloticase responders and $1.04 \pm 0.53$ in subjects receiving placebo. As shown in figure 1 , subjects receiving placebo exhibited a change of $0.26 \pm 0.41$ in the Fib4 score over the six months of the RCTs compared with $0.13 \pm 0.62$ in the pegloticase responders ( $p=0.048$; by linear regression). When only the subjects with a Fib4 value $>1.3$ were considered, a significant difference in the change in the Fib4 values over the 6 months of the trial between pegloticase responders and those receiving placebo was also observed $(-0.15 \pm 0.67$ vs $0.37 \pm 0.42, p=0.004$ by linear regression). The correlations between serum urate area under the curve (AUC) over the 6 months of the trial and the change in Fib4 value was $r_{s}=0.33, p=0.0 .0004$ (Spearman rank-order correlation coefficient). Finally, multiple linear regression analysis indicated serum urate AUC (as a surrogate measure for group) is the main contributor to the change in Fib4 ( $p=0.018$ by linear regression).

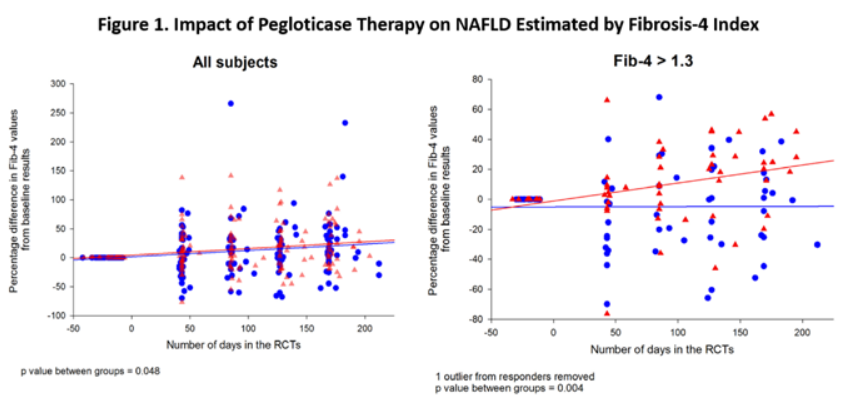

Conclusion: The data are consistent with the conclusion that persistent lowering of serum urate had a significant impact on Fib4 levels, implying a possible effect on the course of NAFLD. The results support a more complete analysis involving biopsy examination of the impact of urate on liver inflammation and fibrosis. References:

[1] Yang C et al. PlosOne2017; 12:e0177249

[2] Jaruvongvanich V et al. Eur J Gastroenterol Hepatol 2017; 29:1031

[3] Jaruvongvanich V et al. Eur J Gastroenterol Hepatol 2017; 29:694

[4] Sundy JS, et al. JAMA. 2011; 306 (7):711-20

[5] Sterling RK et al. Hepatol 2006; 43:1317

[6] Shah AG et al. Clin Gastroenterol Hepatol 2009;7:1104
Disclosure of Interests: : Naomi Schlesinger Grant/research support from: Pfizer, Amgen, Consultant of: Novartis, Horizon Therapeutics, Selecta Biosciences, Olatec, IFM Therapeutics, Mallinckrodt Pharmaceuticals, Anthony Yeo Employee of: Horizon Therapeutics, Peter Lipsky Consultant of: Horizon Therapeutics

DOI: 10.1136/annrheumdis-2020-eular.2360

\section{THU0434 LEFLUNOMIDE CO-THERAPY WITH PEGLOTICASE IN UNCONTROLLED GOUT}

K. Masri ${ }^{1}$, K. Winterling ${ }^{2}$, B. Lamoreaux ${ }^{2} .{ }^{1}$ Bon Secours Rheumatology Center, Richmond, United States of America; ${ }^{2}$ Horizon Therapeutics, Lake Forest, United States of America

Background: Pegloticase is a PEGylated biologic for uncontrolled gout that has well-established efficacy. However, anti-drug antibody (ADA) development causes many patients to discontinue treatment before receiving the full therapeutic course. ${ }^{1}$ ADAs bind to the pegloticase molecule, leading to reduced therapeutic efficacy and discontinuation of therapy. ${ }^{2}$ Emerging data indicates that co-treatment with pegloticase and immunomodulating agents may prevent ADA development, allowing more patients to receive a full course of treatment. Prio case reports describe the results of pegloticase treatment with methotrexate azathioprine or cyclosporine ${ }^{3-6}$; however, the literature does not contain information on the use of leflunomide with pegloticase.

Objectives: To evaluate overall pegloticase responder rate in uncontrolled gout patients co-treated with leflunomide.

Methods: This retrospective study was conducted in a rheumatology practice where an immunomodulatory agent is typically used in conjunction with pegloticase. Patients co-treated with oral leflunomide $(20 \mathrm{mg} /$ day) and pegloticase $(8 \mathrm{mg}$ infusion) were included. Extracted data included demographics, gout characteristics, pegloticase therapy parameters (serum uric acid [sUA], number of infusions), leflunomide therapy parameters (timing with respect to the first pegloticase infusion, dose, route), adverse events (e.g., gout flare, infusion reactions), and safety information (clinical laboratory parameters). Prior to each infusion all patients were administered a standard prophylaxis regimen of fexofenadine the night before and day of infusion, solumedrol day of infusion. The primary outcome was the proportion of pegloticase responders, defined as those receiving $\geq 12$ infusions.

Results: At data collection, 10 patients ( 5 male, $72.7 \pm 12.5$ years old, baseline $\mathrm{sUA}=6.59 \pm 3.15 \mathrm{mg} / \mathrm{dL}$ ) had been co-treated with pegloticase and leflunomide 4 patients $(40 \%)$ received $\geq 12$ infusions of pegloticase; 2 patients began treatment but were lost to follow-up, though neither of these patients experienced a rise in sUA during therapy. Of the 6 patients described, 4 met the primary outcome for a responder and 2 were lost to follow-up, resulting in $4 / 6$ or $66 \%$ response rate. The 4 remaining patients had not reached their $12^{\text {th }}$ infusion at time of data cutoff and were ongoing with a mean of $8.0 \pm 1.6$ infusions (range: 6-10 infusions). No new safety concerns emerged. One patient had 3 gout flares during treatment and 1 patient required emergency care because of loss of consciousness and wooziness prior to pegloticase infusion due to pre-medication with solumedrol. No clinically meaningful laboratory value changes occurred with the exception of a mild and transient ALT rise in 1 patient.

Conclusion: Preliminary evidence suggests that low-to-moderate immunomodulation can minimize or prevent ADA formation against pegloticase and increase the number of patients fully benefitting from pegloticase. No prior studies have examined the effect of leflunomide on pegloticase responder rates. The current study indicates that oral leflunomide may be a viable immunomodulator for patients with uncontrolled gout undergoing pegloticase therapy.

References:

[1] Sundy JS, et al. JAMA, 2011;306(7):711-720

[2] Lipsky PE, et al. Arthritis Res Ther 2014;16:R60.

[3] Botson J and Peterson J. Ann Rheum Dis. 2019; 78: A1289.

[4] Berhanu AA, et al. Semin Arthritis Rheum, 2017;46(6):754-758

[5] Hershfeld MS, et al. Arthritis Res Ther, 2014;16(2):R63

[6] Bessen MY, et al. Int J Clin Rheum, 2019;14(6):238-245

Disclosure of Interests: : Karim Masri Shareholder of: Horizon Therapeutics, Corbus Pharmaceuticals, Gilead, Lineage Cell Therapeutics, Speakers bureau Horizon Therapeutics, Pfizer, Novartis, Lilly, Kevin Winterling Shareholder of: Horizon Therapeutics, Employee of: Horizon Therapeutics, Brian LaMoreaux Shareholder of: Horizon Therapeutics, Employee of: Horizon Therapeutics DOI: 10.1136/annrheumdis-2020-eular.3891

\section{THU0435 \\ CALCIUM PYROPHOSPHATE DIHYDRATE (CPPD) CRYSTALS BUT NOT BASIC CALCIUM PHOSPHATE (BCP) CRYSTALS INDUCE SYNDECAN-4 EXPRESSION IN CARTILAGE}

S. Nasi ${ }^{1}$, J. Bertrand ${ }^{2}$, M. Bollmann ${ }^{2}$, R. Stange ${ }^{3}$, T. Pap $^{3} .{ }^{1}$ University Hospital of Münster, Münster, Germany; ${ }^{2}$ Otto von Guericke University Magdeburg, Magdeburg, Germany; ${ }^{3}$ University Hospital of Münster, Münster, Germany 